\title{
Research on Campus New Facilities Arrangement Based on SLP Method
}

\author{
Yingyi HUANG ${ }^{1,2}$, Jianhong GUO ${ }^{1,2}$ * \\ ${ }^{1}$.TSL School of Busines, Quanzhou Normal University \\ 2. The Cloud Computing \& Internet of Things \& Electronic Commerce University Engineering Center in Fujian Province \\ Quanzhou, China
}

\begin{abstract}
The facility layout has a great influence on teaching activities and entertainment for teachers and students. The appropriate and reasonable campus facility layout is beneficial for the development of school. The paper takes a certain university as a research object and uses SLP from to chart, job related graph method, logistics related graph, etc, to analyze the logistics relation between essentials and facilities on campus. Finally, it gets a comprehensive correlation results among work units. And it can offer academic support for the decision of new facilities layout on campus.
\end{abstract}

\section{Keywords-facility layout; campus; new facilities}

\section{INTRODUCTION}

Whether facilities location is reasonable or not will influence the campus environment construction. And reasonable and scientific campus facilities location is good for constructing favorable campus environment and promotes the sustainable development for the environment. The paper takes a university as an example, combining its features to study its new facilities arrangement.

\section{ANALYSIS OF CAMPUS FACILITIES}

.We get five elements of campus facilities arrangement by combining SLP method and campus features, such as people, quantity, routine, structure, time. Firstly, as shown in Fig. 1, we drew a campus plan so that we can know the relative position of campus facilities. Then used map to measure the distance between each two location points on the basis of campus plan. Finally, a traffic from to chart is given based on the assumption of flow among campus facilities, as seen in the following in TABLE I.

TABLE I. TRAFFIC FROM TO CHART (UNIT:METERS)

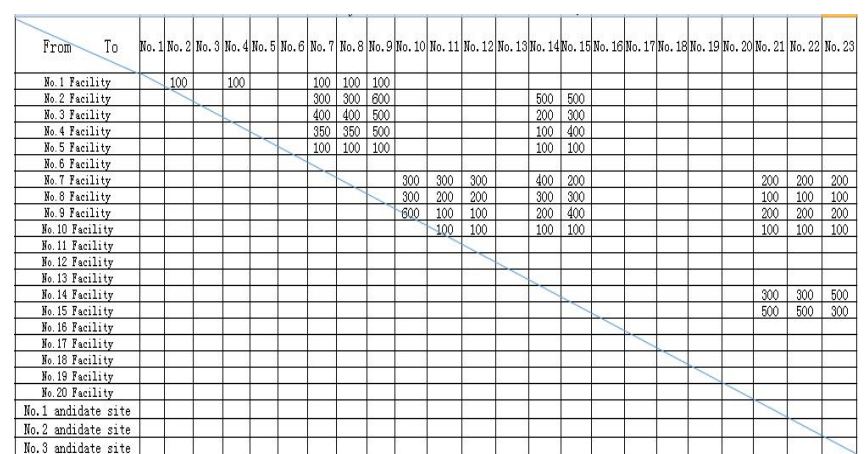

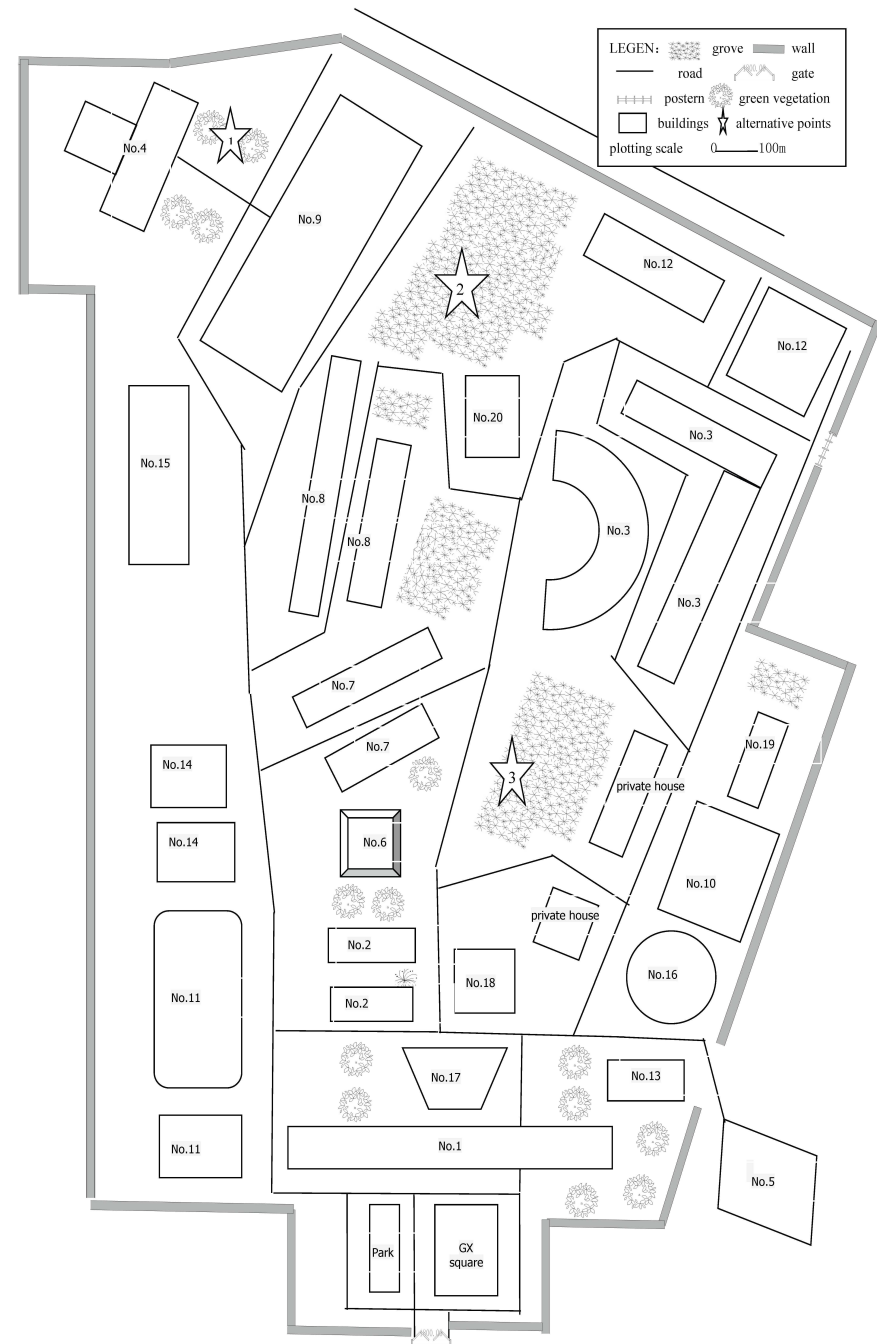

Fig. 1. Campus plan.

\section{ANALYSIS OF NEW FACILITIES ARRANGEMENT}

\section{A. Logistics intension from to chart}

Logistics intension is the sum of the product of the traffic and distance. We calculated the logistics intension of facilities according to TABLE $\mathrm{I}$ and the assumption of flow among campus facilities, as seen in the following in TABLE II. 


\section{B. Logistics related graph}

It can reveal the grade of logistics intension base on TABLE II. Then obtain the proportion of each logistics intension grade (see TABLE III) by combining the famous "A,E,I,O,U” hierarchy classification method. And also can get a logistics intension related graph, just seen in the following Fig. 2.

TABLE II. LOGISTICS INTENSION CORRELATION TABLE OF FACILITIES

\begin{tabular}{|c|c|c|c|c|c|c|c|c|c|c|c|}
\hline No. & Route & Logistics Intension & Grade & No. & Route & Logistics Intension & Grade & No. & Route & Logistics Intension & Grade \\
\hline 1 & $3-9$ & 750 & $\mathrm{~A}$ & 22 & $10-22$ & 130 & $\mathrm{I}$ & 43 & $2-14$ & 75 & $\mathrm{O}$ \\
\hline 2 & $9-10$ & 720 & A & 23 & $10-21$ & 130 & I & 44 & $8-23$ & 73 & $\mathrm{O}$ \\
\hline 3 & $2-9$ & 396 & A & 24 & $2-8$ & 123 & $\mathrm{I}$ & 45 & $5-14$ & 71 & $\mathrm{O}$ \\
\hline 4 & $3-8$ & 360 & $\mathrm{~A}$ & 25 & $8-11$ & 122 & $\mathrm{I}$ & 46 & $1-8$ & 71 & $\mathrm{O}$ \\
\hline 5 & $3-15$ & 360 & A & 26 & $5-9$ & 120 & I & 47 & $7-15$ & 70 & $\mathrm{O}$ \\
\hline 6 & $3-7$ & 356 & A & 27 & $7-22$ & 116 & $\mathrm{I}$ & 48 & $10-14$ & 69 & $\mathrm{O}$ \\
\hline 7 & $14-23$ & 300 & A & 28 & $15-21$ & 115 & I & 49 & $4-14$ & 68 & $\mathrm{O}$ \\
\hline 8 & $8-10$ & 288 & $\mathrm{E}$ & 29 & $4-9$ & 110 & I & 50 & $9-22$ & 64 & $\mathrm{O}$ \\
\hline 9 & $2-15$ & 280 & $\mathrm{E}$ & 30 & $1-4$ & 110 & I & 51 & $10-11$ & 61 & $\mathrm{O}$ \\
\hline 10 & $15-23$ & 264 & $\mathrm{E}$ & 31 & $7-21$ & 108 & I & 52 & $1-7$ & 54 & $\mathrm{O}$ \\
\hline 11 & $7-10$ & 237 & $E$ & 32 & 4-15 & 108 & I & 53 & $10-12$ & 51 & $\mathrm{O}$ \\
\hline 12 & $7-12$ & 225 & $E$ & 33 & $7-23$ & 104 & $\mathrm{I}$ & 54 & $10-23$ & 49 & $\mathrm{O}$ \\
\hline 13 & 4-7 & 217 & $E$ & 34 & $9-14$ & 100 & $\mathrm{I}$ & 55 & $8-22$ & 48 & $\mathrm{O}$ \\
\hline 14 & $9-23$ & 192 & $E$ & 35 & $1-9$ & 100 & $\mathrm{I}$ & 56 & $8-21$ & 45 & $\mathrm{O}$ \\
\hline 15 & 4-8 & 186 & $E$ & 36 & $5-8$ & 97 & $\mathrm{I}$ & 57 & $9-15$ & 36 & $\mathrm{O}$ \\
\hline 16 & 3-14 & 186 & $E$ & 37 & $9-11$ & 85 & $\mathrm{I}$ & 58 & $7-14$ & 36 & $\mathrm{O}$ \\
\hline 17 & $14-22$ & 186 & $\mathrm{E}$ & 38 & $5-7$ & 80 & $\mathrm{O}$ & 59 & $9-21$ & 32 & $\mathrm{O}$ \\
\hline 18 & $14-21$ & 174 & $\mathrm{E}$ & 39 & 8-15 & 78 & $\mathrm{O}$ & 60 & $1-2$ & 28 & $\mathrm{O}$ \\
\hline 19 & $8-12$ & 152 & $\mathrm{E}$ & 40 & 8-14 & 78 & $\mathrm{O}$ & 61 & 5-15 & 11 & $\mathrm{O}$ \\
\hline 20 & $15-22$ & 150 & I & 41 & $9-12$ & 76 & $\mathrm{O}$ & 62 & $10-15$ & 11 & $\mathrm{O}$ \\
\hline 21 & $7-11$ & 132 & I & 42 & $2-7$ & 75 & $\mathrm{O}$ & & & & \\
\hline
\end{tabular}

\section{Analysis of non-logistics factors of campus facilities}

In addition, there is still non-logistics relation among campus facilities. We generally adopt qualitative methods to analyze these factors. According to grading reasons(factors of noise, security, life service, campus large-scale activities, administration, entertainment, etc, are chosen in this paper),we provide relation grades of A,E,I,O,U and X6 qualitatively, and draw a non-logistics related graph among campus facilities in Fig. 3, and got non-logistics intension of campus facilities(see TABLE IV ).In related graph, each rhombus unit reflects relation grade between each two facilities. And the codes in this figure are grading reasons.

\section{Comprehensive analysis of corrected campus facilities}

Logistics and non-logistics factors have an effect on campus facilities location to each other. We can mark off a logistics intension to determine campus facilities location to each other through synthesizing two factors, and considering the relative importance of logistics and non-logistics factors. And it can use the ratio $m: n$ to describe When elative these importance. Accordingly, we set the ratio $m: n$ of relative importance on logistics and non-logistics factors equal to $3: 1$.

TABLE III. THE PROPORTION OF LOGISTICS INTENSION GRADE

\begin{tabular}{|l|c|c|c|}
\hline $\begin{array}{l}\text { Logistics } \\
\text { Intension grade }\end{array}$ & Symbol & $\begin{array}{c}\text { The Proportion of } \\
\text { Logistics } \\
\text { Route(\%) }\end{array}$ & $\begin{array}{c}\text { The Proportion of } \\
\text { Logistics } \\
\text { Volume(\%) }\end{array}$ \\
\hline $\begin{array}{l}\text { Ultrahigh } \\
\text { Logistics Intension }\end{array}$ & A & 11 & 35 \\
\hline
\end{tabular}

\begin{tabular}{|l|c|c|c|}
\hline \multicolumn{4}{|c|}{ Cont. to TABLEIII } \\
\hline $\begin{array}{l}\text { High Logistics } \\
\text { Intension }\end{array}$ & E & 19 & 28 \\
\hline $\begin{array}{l}\text { Relative High } \\
\text { Logistics Intension }\end{array}$ & I & 29 & 22 \\
\hline $\begin{array}{l}\text { Normal High } \\
\text { Logistics Intension }\end{array}$ & O & 41 & 15 \\
\hline Ignorable Transition & U & - & - \\
\hline
\end{tabular}

According to this ratio, we evaluate the grades in TABLE II and TABLE IV, and choose $\mathrm{A}=4, \mathrm{E}=3, \mathrm{I}=2, \mathrm{O}=1, \mathrm{U}=0, \mathrm{X}=$ 1.When weighted calculating the logistics and non-logistics factors on the basis of the ratio $3: 1$, the calculating results are shown in TABLE $\mathrm{V}$ and TABLE VI.

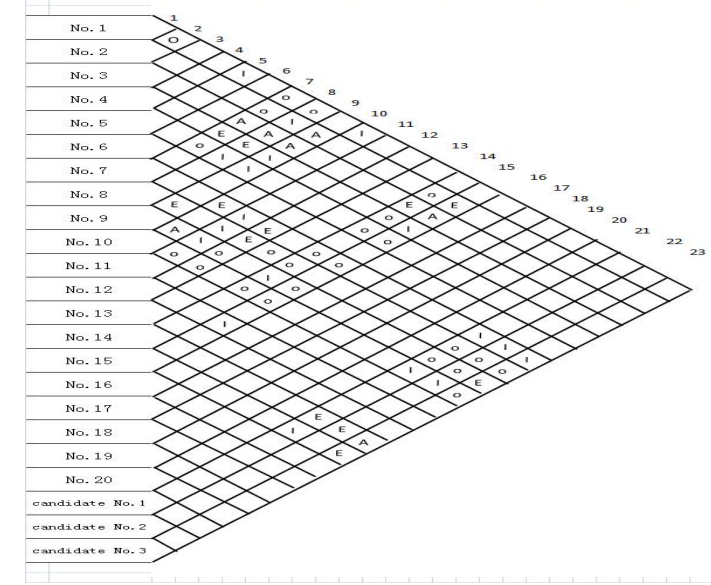

Fig. 2. Logistics intension related graph of campus facilities. 
TABLE IV. NON-LOGISTICS INTENSION OF CAMPUS FACILITIES

\begin{tabular}{|c|c|c|c|}
\hline No. & Work Unit Pair (Logistics Route) & Non-logistics closeness grade & Reason's Number \\
\hline 1 & $1-11$ & $\mathrm{X}$ & 1 \\
\hline 2 & $1-12$ & $\mathrm{X}$ & 1 \\
\hline 3 & $1-13$ & $\mathrm{I}$ & 5 \\
\hline 4 & $2-11$ & $\mathrm{X}$ & 1 \\
\hline 5 & $2-12$ & $\mathrm{X}$ & 1 \\
\hline 6 & $2-13$ & I & 5 \\
\hline 7 & $3-11$ & $\mathrm{X}$ & 12 \\
\hline 8 & $3-12$ & $\mathrm{X}$ & 12 \\
\hline 9 & $3-13$ & I & 5 \\
\hline 10 & $3-14$ & $\mathrm{X}$ & 12 \\
\hline 11 & $3-15$ & $\mathrm{X}$ & 12 \\
\hline 12 & $4-11$ & $\mathrm{X}$ & 1 \\
\hline 13 & $4-12$ & $X$ & 1 \\
\hline 14 & $4-13$ & I & 5 \\
\hline 15 & $5-11$ & $X$ & 1 \\
\hline 16 & $5-12$ & $X$ & 1 \\
\hline 17 & $5-13$ & I & 5 \\
\hline 18 & $6-13$ & I & 5 \\
\hline 19 & $7-11$ & I & 46 \\
\hline 20 & $7-12$ & I & 46 \\
\hline 21 & $7-14$ & $\mathrm{E}$ & 3 \\
\hline 22 & $7-15$ & $\mathrm{E}$ & 3 \\
\hline 23 & $8-11$ & I & 46 \\
\hline 24 & $8-12$ & I & 46 \\
\hline 25 & $8-14$ & $\mathrm{E}$ & 43 \\
\hline 26 & $8-15$ & $E$ & 3 \\
\hline 27 & $9-11$ & I & 46 \\
\hline 28 & $9-12$ & I & 46 \\
\hline 29 & $9-14$ & $\mathrm{E}$ & 3 \\
\hline 30 & $9-15$ & $\mathrm{E}$ & 3 \\
\hline 31 & $10-11$ & $\mathrm{X}$ & 1 \\
\hline 32 & $10-12$ & $X$ & 2 \\
\hline 33 & $11-21$ & I & 46 \\
\hline 34 & $11-22$ & I & 46 \\
\hline 35 & $11-23$ & I & 46 \\
\hline 36 & $12-21$ & I & 6 \\
\hline 37 & $12-22$ & I & 6 \\
\hline 38 & $12-23$ & I & 6 \\
\hline 39 & $14-21$ & $\mathrm{E}$ & 3 \\
\hline 40 & $14-22$ & $E$ & 3 \\
\hline 41 & $14-23$ & $\mathrm{E}$ & 3 \\
\hline 42 & $15-21$ & $E$ & 3 \\
\hline 43 & $15-22$ & $\mathrm{E}$ & 3 \\
\hline 44 & $15-23$ & $\mathrm{E}$ & 3 \\
\hline
\end{tabular}

\section{E. Determining campus new facilities location}

The location of campus new facilities will be chosen among 3 uncertain locations. On the basis of TABLE VI, we calculate the sum of the comprehensive related score of work unit pairs of uncertain locations 1,2,3, and get the TABLE VII. 


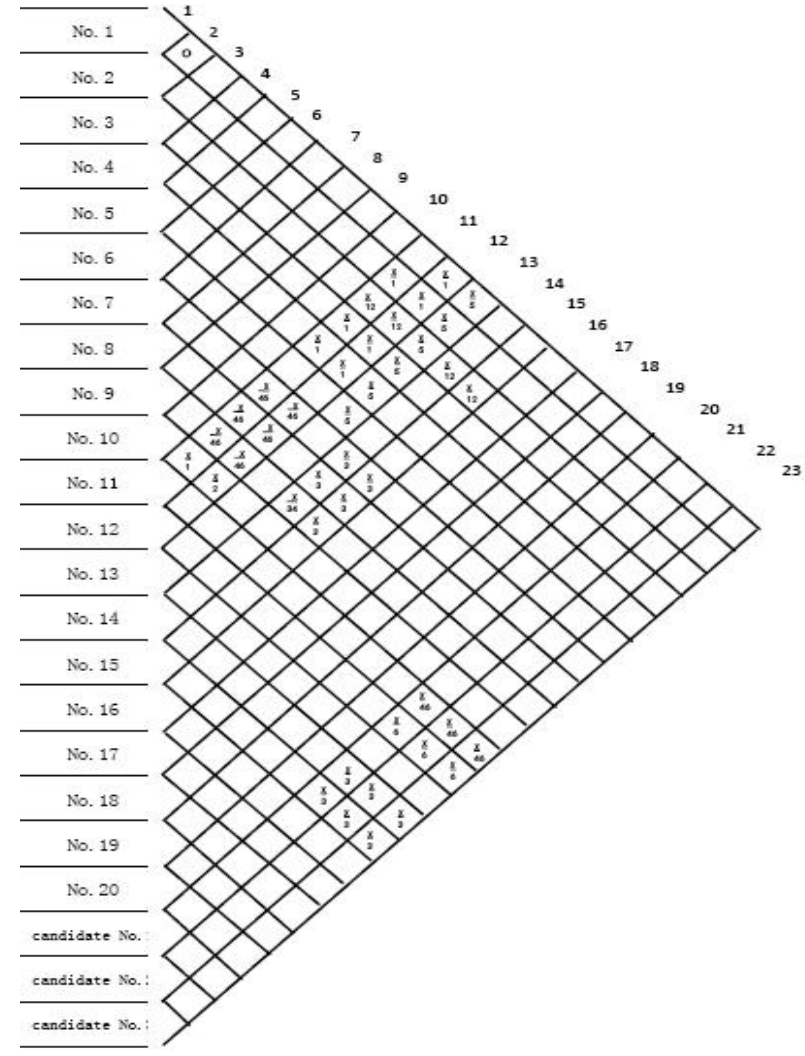

Fig. 3. Non-logistics related graph of campus facilities.

The higher of the comprehensive relation score of facilities is, the closer between this facility and other facilities is. Because uncertain location 3's comprehensive relation score is the highest, the location of campus new teaching building is in uncertain location 3 .

TABLE V. COMPREHENSIVELY CORRELATION OF CAMPUS FACILITIES

\begin{tabular}{|c|c|c|c|c|c|c|c|}
\hline \multirow{2}{*}{ No. } & \multirow{2}{*}{$\begin{array}{c}\text { Work } \\
\text { Unit Pair }\end{array}$} & $\begin{array}{c}\text { Cogistics } \\
\text { Factor(Weight } \\
\text { value:3) }\end{array}$ & \multicolumn{2}{c|}{$\begin{array}{c}\text { Non-logistics } \\
\text { Factor(Weight } \\
\text { value:1) }\end{array}$} & \multicolumn{2}{c|}{$\begin{array}{c}\text { Comprehensive } \\
\text { correlation }\end{array}$} \\
\cline { 3 - 7 } & & Grade & Score & Grade & Score & Score & Grade \\
\hline 1 & $1-2$ & $\mathrm{O}$ & 1 & $\mathrm{U}$ & 0 & 3 & $\mathrm{O}$ \\
\hline 2 & $1-4$ & $\mathrm{I}$ & 2 & $\mathrm{U}$ & 0 & 6 & $\mathrm{I}$ \\
\hline 3 & $1-7$ & $\mathrm{O}$ & 1 & $\mathrm{U}$ & 0 & 3 & $\mathrm{O}$ \\
\hline 4 & $1-8$ & $\mathrm{O}$ & 1 & $\mathrm{U}$ & 0 & 3 & $\mathrm{O}$ \\
\hline 5 & $1-9$ & $\mathrm{I}$ & 2 & $\mathrm{X}$ & -1 & -1 & $\mathrm{X}$ \\
\hline 6 & $1-11$ & $\mathrm{U}$ & 0 & $\mathrm{X}$ & -1 & -1 & $\mathrm{X}$ \\
\hline 7 & $1-12$ & $\mathrm{U}$ & 0 & $\mathrm{I}$ & 2 & 2 & $\mathrm{O}$ \\
\hline 8 & $2-7$ & 0 & 1 & $\mathrm{U}$ & 0 & 3 & $\mathrm{O}$ \\
\hline 9 & $2-8$ & $\mathrm{I}$ & 2 & $\mathrm{U}$ & 0 & 6 & $\mathrm{I}$ \\
\hline 10 & $2-9$ & $\mathrm{~A}$ & 4 & $\mathrm{U}$ & 0 & 12 & $\mathrm{~A}$ \\
\hline 11 & $2-11$ & $\mathrm{U}$ & 0 & $\mathrm{X}$ & -1 & -1 & $\mathrm{X}$ \\
\hline 12 & $2-12$ & $\mathrm{U}$ & 0 & $\mathrm{X}$ & -1 & -1 & $\mathrm{X}$ \\
\hline 13 & $2-13$ & $\mathrm{U}$ & 0 & $\mathrm{I}$ & 2 & 2 & $\mathrm{O}$ \\
\hline 14 & $2-14$ & $\mathrm{O}$ & 1 & $\mathrm{U}$ & 0 & 3 & $\mathrm{O}$ \\
\hline 15 & $2-15$ & $\mathrm{E}$ & 3 & $\mathrm{U}$ & 0 & 9 & $\mathrm{E}$ \\
\hline 16 & $3-7$ & $\mathrm{~A}$ & 4 & $\mathrm{U}$ & 0 & 12 & $\mathrm{~A}$ \\
\hline 17 & $3-8$ & $\mathrm{~A}$ & 4 & $\mathrm{U}$ & 0 & 12 & $\mathrm{~A}$ \\
\hline
\end{tabular}

\begin{tabular}{|c|c|c|c|c|c|c|c|}
\hline \multicolumn{8}{|c|}{ Cont.to TABLE V } \\
\hline 18 & $3-9$ & A & 4 & $\mathrm{U}$ & 0 & 12 & A \\
\hline 19 & $3-11$ & $\mathrm{U}$ & 0 & $\mathrm{X}$ & -1 & -1 & $\mathrm{X}$ \\
\hline 20 & $3-12$ & $\mathrm{U}$ & 0 & $\mathrm{X}$ & -1 & -1 & $\mathrm{X}$ \\
\hline 21 & $3-13$ & $\mathrm{U}$ & 0 & I & 2 & 2 & $\mathrm{O}$ \\
\hline 22 & $3-14$ & $\mathrm{O}$ & 1 & $\mathrm{X}$ & -1 & 2 & $\mathrm{O}$ \\
\hline 23 & $3-15$ & I & 2 & $\mathrm{X}$ & -1 & 5 & I \\
\hline 24 & $4-7$ & $\mathrm{E}$ & 3 & $\mathrm{U}$ & 0 & 9 & $\mathrm{E}$ \\
\hline 25 & $4-8$ & E & 3 & $\mathrm{U}$ & 0 & 9 & E \\
\hline 26 & $4-9$ & I & 2 & $\mathrm{U}$ & 0 & 6 & E \\
\hline 27 & $4-11$ & $\mathrm{U}$ & 0 & $\mathrm{X}$ & -1 & -1 & $\mathrm{X}$ \\
\hline 28 & $4-12$ & $\mathrm{U}$ & 0 & $\mathrm{X}$ & -1 & -1 & $\mathrm{X}$ \\
\hline 29 & $4-13$ & $\mathrm{U}$ & 0 & I & 2 & 2 & $\mathrm{O}$ \\
\hline 30 & 4-14 & $\mathrm{E}$ & 3 & $\mathrm{U}$ & 0 & 9 & $\mathrm{E}$ \\
\hline 31 & $4-15$ & A & 4 & $\mathrm{U}$ & 0 & 12 & A \\
\hline 32 & $5-7$ & $\mathrm{O}$ & 1 & $\mathrm{U}$ & 0 & 3 & $\mathrm{O}$ \\
\hline 33 & $5-8$ & I & 2 & U & 0 & 6 & I \\
\hline 34 & 5-9 & I & 2 & $\mathrm{U}$ & 0 & 6 & I \\
\hline 35 & $5-11$ & $\mathrm{U}$ & 0 & $X$ & -1 & -1 & $X$ \\
\hline 36 & $5-12$ & $\mathrm{U}$ & 0 & $X$ & -1 & -1 & $X$ \\
\hline 37 & $5-13$ & $\mathrm{U}$ & 0 & $\mathrm{I}$ & 2 & 2 & $\mathrm{O}$ \\
\hline 38 & $5-14$ & $\mathrm{O}$ & 1 & U & 0 & 3 & $\mathrm{O}$ \\
\hline 39 & $5-15$ & $\mathrm{O}$ & 1 & $\mathrm{U}$ & 0 & 3 & $\mathrm{O}$ \\
\hline 40 & 6-13 & $\mathrm{U}$ & 0 & $\mathrm{I}$ & 2 & 6 & I \\
\hline 41 & $7-10$ & $\mathrm{E}$ & 3 & $\mathrm{U}$ & 0 & 9 & $\mathrm{E}$ \\
\hline 42 & $7-11$ & I & 2 & I & 2 & 8 & E \\
\hline 43 & $7-12$ & $\mathrm{E}$ & 3 & $\mathrm{I}$ & 2 & 11 & $\mathrm{E}$ \\
\hline 44 & $7-14$ & $\mathrm{O}$ & 1 & $\mathrm{E}$ & 3 & 6 & $\mathrm{I}$ \\
\hline 45 & $7-15$ & $\mathrm{O}$ & 1 & $\mathrm{E}$ & 3 & 6 & I \\
\hline 46 & $7-21$ & $\mathrm{I}$ & 2 & $\mathrm{U}$ & 0 & 6 & I \\
\hline 47 & $7-22$ & $\mathrm{I}$ & 2 & $\mathrm{U}$ & 0 & 6 & I \\
\hline 48 & $7-23$ & $\mathrm{I}$ & 2 & $\mathrm{U}$ & 0 & 6 & I \\
\hline 49 & $8-10$ & $\mathrm{E}$ & 3 & $\mathrm{U}$ & 0 & 9 & E \\
\hline 50 & $8-11$ & I & 2 & I & 2 & 8 & E \\
\hline 51 & $8-12$ & $\mathrm{E}$ & 3 & I & 2 & 11 & $\mathrm{E}$ \\
\hline 52 & $8-14$ & $\mathrm{O}$ & 1 & $\mathrm{E}$ & 3 & 6 & I \\
\hline 53 & $8-15$ & $\mathrm{O}$ & 1 & $\mathrm{E}$ & 3 & 6 & I \\
\hline 54 & $8-21$ & $\mathrm{O}$ & 1 & $\mathrm{U}$ & 0 & 3 & $\mathrm{O}$ \\
\hline 55 & $8-22$ & $\mathrm{O}$ & 1 & $\mathrm{U}$ & 0 & 3 & $\mathrm{O}$ \\
\hline 56 & $8-23$ & $\mathrm{O}$ & 1 & $\mathrm{U}$ & 0 & 3 & $\mathrm{O}$ \\
\hline 57 & $9-10$ & $\mathrm{~A}$ & 4 & $\mathrm{U}$ & $\mathrm{O}$ & 12 & A \\
\hline 58 & $9-11$ & I & 2 & I & 2 & 8 & E \\
\hline 59 & $9-12$ & $\mathrm{O}$ & 1 & $\mathrm{I}$ & 2 & 5 & I \\
\hline 60 & $9-14$ & $\mathrm{I}$ & 2 & $\mathrm{E}$ & 3 & 9 & $\mathrm{E}$ \\
\hline 61 & $9-15$ & $\mathrm{O}$ & 1 & $E$ & 3 & 6 & I \\
\hline 62 & $9-21$ & $\mathrm{O}$ & 1 & $\mathrm{U}$ & 0 & 3 & $\mathrm{O}$ \\
\hline 63 & $9-22$ & $\mathrm{O}$ & 1 & $\mathrm{U}$ & 0 & 3 & $\mathrm{O}$ \\
\hline 64 & $9-23$ & $\mathrm{E}$ & 3 & $\mathrm{U}$ & 0 & 9 & $\mathrm{E}$ \\
\hline 65 & $10-11$ & $\mathrm{O}$ & 1 & $\mathrm{X}$ & -1 & 2 & $\mathrm{O}$ \\
\hline 66 & $10-12$ & $\mathrm{O}$ & 1 & $X$ & -1 & 2 & $\mathrm{O}$ \\
\hline 67 & $10-14$ & $\mathrm{O}$ & 1 & $\mathrm{U}$ & 0 & 3 & $\mathrm{O}$ \\
\hline 68 & $10-15$ & $\mathrm{O}$ & 1 & $\mathrm{U}$ & 0 & 3 & $\mathrm{O}$ \\
\hline 69 & $10-21$ & I & 2 & $\mathrm{U}$ & 0 & 6 & I \\
\hline 70 & $10-22$ & $\mathrm{I}$ & 2 & $\mathrm{U}$ & 0 & 6 & I \\
\hline 71 & $10-23$ & $\mathrm{O}$ & 1 & $\mathrm{U}$ & 0 & 3 & $\mathrm{O}$ \\
\hline 72 & $11-21$ & $\mathrm{U}$ & 0 & $\mathrm{E}$ & 3 & 3 & $\mathrm{O}$ \\
\hline 73 & $11-22$ & $\mathrm{U}$ & 0 & $\mathrm{E}$ & 3 & 3 & $\mathrm{O}$ \\
\hline 74 & $11-23$ & $\mathrm{U}$ & 0 & $\mathrm{E}$ & 3 & 3 & $\mathrm{O}$ \\
\hline 75 & $12-21$ & $\mathrm{U}$ & 0 & E & 3 & 3 & $\mathrm{O}$ \\
\hline
\end{tabular}




\begin{tabular}{|c|c|c|c|c|c|c|c|}
\hline \multicolumn{7}{|c|}{ Cont.to TABLE V } \\
\hline 76 & $12-22$ & $\mathrm{U}$ & 0 & $\mathrm{E}$ & 3 & 3 & $\mathrm{O}$ \\
\hline 77 & $12-23$ & $\mathrm{U}$ & 0 & $\mathrm{E}$ & 3 & 3 & $\mathrm{O}$ \\
\hline 78 & $14-21$ & $\mathrm{E}$ & 3 & $\mathrm{E}$ & 3 & 12 & $\mathrm{~A}$ \\
\hline 79 & $14-22$ & $\mathrm{E}$ & 3 & $\mathrm{E}$ & 3 & 12 & $\mathrm{~A}$ \\
\hline 80 & $14-23$ & $\mathrm{~A}$ & 4 & $\mathrm{E}$ & 3 & 15 & $\mathrm{~A}$ \\
\hline 81 & $15-21$ & $\mathrm{I}$ & 2 & $\mathrm{E}$ & 3 & 9 & $\mathrm{E}$ \\
\hline 82 & $15-22$ & $\mathrm{I}$ & 2 & $\mathrm{E}$ & 3 & 9 & $\mathrm{E}$ \\
\hline 83 & $15-23$ & $\mathrm{E}$ & 3 & $\mathrm{E}$ & 3 & 12 & $\mathrm{~A}$ \\
\hline
\end{tabular}

TABLE VI. CALCULATION RESULTS OF GRADING DIVIDING

\begin{tabular}{|c|c|c|c|}
\hline $\begin{array}{c}\text { The Number of } \\
\text { Work Unit Pair }\end{array}$ & Percentage(\%) & Sum & Correlation Grade \\
\hline 10 & $4.00 \%$ & $12-15$ & A \\
\hline 15 & $6.00 \%$ & $8-11$ & E \\
\hline 18 & $7.00 \%$ & $5-7$ & I \\
\hline 30 & $12.00 \%$ & $1-4$ & O \\
\hline 170 & $67.00 \%$ & $0-1$ & U \\
\hline 10 & $4.00 \%$ & -1 & X \\
\hline 253 & $100 \%$ & \multicolumn{2}{|c}{ Sum } \\
\hline
\end{tabular}

TABLE VII. THE COMPREHENSIVE RELATION SCORE OF UNCERTAIN LOCATIONS

\begin{tabular}{|c|c|}
\hline Facility & The Sum of Comprehensive Relation Score \\
\hline Uncertain Location 1 & 66 \\
\hline Uncertain Location 2 & 67 \\
\hline Uncertain Location 3 & 77 \\
\hline
\end{tabular}

\section{CONCLUSION}

The link between campus facilities layout and environment construction is close. Reasonable and scientific campus new facilities layout will make full use of new facilities functions and promote the sustainable development of campus environment. And favorable campus environment is beneficial for holding teachers' and students' teaching activities and recreational activities, increasing students' satisfaction to campus, and facilitating campus development.

This paper combining the actual situation of a certain college assumes three facility points as the uncertain locations and uses SLP method to firstly analyze and input data of five SLP elements on campus, to draw the campus plan, traffic and distance from to chart, then to analyze the logistics and nonlogistics relation of campus facilities by using these basic data and from to chart, related graph and A,E,I,O,U hierarchy classification method, finally to choose 3 uncertain location as the ultimate location by generally considering the comprehensive relation between uncertain locations and other facility locations. In this paper, decision support is provided for campus new facility layout problem.

\section{ACKNOWLEDGMENT}

This work is partially supported by the Natural Science Foundation of Fujian Province for Youths, China (grant No. 2017J05116), the Natural Science Foundation of Fujian Province, China (grant No. 2015J01286), the JK class project in Fujian Province Department of Education (grant No. JK2014037), the Education Research Project of Fujian Province for Young and Middle-aged Teacher (grant No. JAS150448). We deeply appreciate the organizations mentioned above.

\section{REFERENCES}

[1] Q. Li,Z.R. Li . "Analysis and design of logistics facilities layout in workshop," Modern manufacturing engineering, vol. 31, pp. 67-70, 2011

[2] N.B. Bridge. "Challenges in the development of environmental management systems onthe modem university campus," Journal of Sustainability in HigherEducation, vol. 13, pp. 133-149, 2012

[3] Z.J. Ren, Z.Y. Liu, X. He. "Research on the Campus Fire Resource Allocation Based on SLP Method," Modern Management, vol. 07, pp. 312-318, 2017.

[4] J.H. Yang. "Design on Layout Rearrangement of Plant Logistics Based on the Combination of SLP and SHA," Applied Mechanics and Materials, vol. 58, pp. 932-937, 2011.

[5] Y. Xiao,Y. K. Cheng,Q.H. Jia. "Application of SLP in Plant Layout Based on Low Carbon Logistics," Advanced Materials Research, vol. 424, pp. 314-317, 2012.

[6] C. Wu,T.J. Hu, X.F. Wang, C. Zheng. "Study on the Functional Zones Layout of Fresh Food Distribution Center Based on the SLP Method," Advanced Materials Research, vol. 694, pp. 3614-3617, 2013.

[7] X.W. Qian. "The Application of SLP with Hungarian Method in the Spatial Layout Planning of Logistics Park-with Chengdu International Railway Container Logistics Park as an example," Advanced Materials Research, vol. 838, pp. 1273-1280, 2014. 